\title{
Seed-mediated Growth of Silver Nanoplates and Investigation on Their Nonlinear Optical Behaviors
}

\author{
Yalan Wang ${ }^{1,2, \dagger}$, Xiang Li ${ }^{1,3, \dagger}$, Anle Wang ${ }^{1}$, Jin Zhang ${ }^{1}$, Jianghai Wo ${ }^{1}$, Ququan Wang ${ }^{2, *}$ \\ ${ }^{1}$ Air Force Early Warning Academy, Wuhan, China \\ ${ }^{2}$ Department of Physics, Wuhan University, Wuhan, China \\ ${ }^{3}$ Hubei Key Laboratory of Ferroelectric and Dielectric Materials and Devices, Faculty of Physics and Electronic Science, Hubei University, \\ Wuhan, China
}

Email address:

qqwang@whu.edu.cn (Ququan Wang)

${ }^{*}$ Corresponding author

$\dagger$ Yalan Wang and Xiang Li are co-first authors.

\section{To cite this article:}

Yalan Wang, Xiang Li, Anle Wang, Jin Zhang, Jianghai Wo, Ququan Wang. Seed-mediated Growth of Silver Nanoplates and Investigation on Their Nonlinear Optical Behaviors. American Journal of Optics and Photonics. Vol. 9, No. 1, 2021, pp. 18-22.

doi: 10.11648/j.ajop.20210901.13

Received: February 23, 2021; Accepted: March 9, 2021; Published: March 26, 2021

\begin{abstract}
Silver nanoplates have obvious advantages compared with other silver structural nanoparticles due to their wide tunability of the local surface plasmon resonance (LSPR) and significant local field enhancement around the sharp corners. Nonlinear optical absorption (NLA) and nonlinear optical refraction (NLR) are widely employed to characterize the nonlinear optical properties due to their simply testing requirement and comparable valuable results. Z-scan method is widely used in optical characterization of nonlinear properties of different materials. Here, a seed-mediated growth method of the silver nanoplates with well controlled size and local surface plasmon wavelength is presented. The nanoparticle size increases gradually and becomes more uniform distribution as the red-shift of the plasmon resonance wavelength from $650 \mathrm{~nm}$ to 950 $\mathrm{nm}$. In particular, we show the dependence of NLA and NLR of the silver nanoplates on the LSPR. The absolute value of effective NLA coefficient increases from 1.38 to $4 \mathrm{~cm} / \mathrm{GW}$ and that of the NLR index increases as large as 3 times when the LSPR wavelength changes from $728 \mathrm{~nm}$ to $898 \mathrm{~nm}$, which is attributed to the strong plasmon absorption and local filed enhancement of larger size silver nanoplates. These findings have great potentials in the explorations of functional non-linear devices.
\end{abstract}

Keywords: Surface Plasmon, Z-scan, Nonlinear Optical, Silver Nanoplates

\section{Introduction}

Plasmon, which describes collective oscillation of electrons on the metal surface, has potential applications in plasmon enhanced photoluminescence [1-3], Raman scattering $[4,5]$, and plasmon enhanced light absorption [68]. Aside from the nanoparticle materials, the plasmon resonance wavelength is strongly dependent on the physical geometrical shape, size and morphology [9-13]. Among the metal nanoparticles plasmonic materials, silver is one of the best candidates due to its lower optical loss in the visible and near infrared ranges. Silver nanoplates have obvious advantages compared with other silver structural nanoparticles due to their wide tunability of the local surface plasmon resonance (LSPR) and significant local field enhancement around the sharp corners, which is attributed to their higher aspect ratio between the two in-plane dipolar and the quadrupolar plasmon modes [14-22].

Nonlinear optical absorption (NLA) and nonlinear optical refraction (NLR) are widely employed to characterize the nonlinear optical properties due to their simply testing requirement and comparable valuable results. Z-scan method is widely used in optical characterization of nonlinear properties of different materials. The nonlinear properties of the metal nanoparticles have attracted much attention in recent years due to their potential application in biology 
sensors and many other areas. Huge works focus on the nonlinear properties of different composite nanoparticles, such as metal nanoparticles with various kinds of geometric shape, metal-semiconductor nanocomposite film and many other plasmon doped materials [23-33].

Here a seed-mediated synthesis method is adopted to fabricate silver nanoplates with widely tunable longitude LSPR wavelength ranging from $650 \mathrm{~nm}$ to $950 \mathrm{~nm}$. A systematical investigation on the NLA and NLR dependence on the LSPR of the silver nanoplates is displayed. The NLR and NLA coefficients of silver nanoplates increase with the red-shift of LSPR, which is attributed to the increase of the strong plasmon absorption and local filed enhancement accompanied with the growing size of silver nanoplates. The absolute value of effective NLA coefficient $|\beta|$ increases from 1.38 to $4 \mathrm{~cm} / \mathrm{GW}$ and that of NLR index $|\gamma|$ rises from 1.4 to $3.71 \mathrm{~cm}^{2} / \mathrm{GW}$. These findings have great potentials in the explorations of functional non-linear devices.

\section{Experimental}

A two-step seed-mediated growth method was applied to synthesize the silver nanoplatelets. In the first step, the suspension of seeds was prepared as follows: $147 \mathrm{ml}$ distilled water was mixed with $9 \mathrm{ml}$ of sodium citrate $\left(\mathrm{Na}_{3} \mathrm{CA}\right)(30$ $\mathrm{mmol} / \mathrm{ml}$ ) under magnetic stirring. Then, $9 \mathrm{ml}$ polyvinyl pyrrolidone (MW 40000, $20.3 \mathrm{mg} / \mathrm{ml}$ ), $0.36 \mathrm{ml}$ hydrogen peroxide $(30 \%), 3 \mathrm{ml}$ silver nitrate $\left(\mathrm{AgNO}_{3}\right)(0.85 \mathrm{mg} / \mathrm{ml})$, and $1.5 \mathrm{ml}$ sodium borohydride $(3.78 \mathrm{mg} / \mathrm{ml})$ were added into the solution in sequence. The second step was beginning with taking out $10 \mathrm{ml}$ as-prepared silver nanoplate seeds into a flask. Then $10 \mathrm{ml}$ of aqueous solution containing Lascorbic acid $(1.2 \mathrm{mmol} / \mathrm{ml})$ and $\mathrm{Na}_{3} \mathrm{CA}(0.4 \mathrm{mmol} / \mathrm{ml})$ were inserted into the seeds under magnetic stirring. Following was the injection of $\mathrm{AgNO}_{3}$ solution $(0.6 \mathrm{mmol} / \mathrm{ml})$ using a syringe pump at a rate of $10 \mathrm{~mL} / \mathrm{h}$. The silver nanoplates with different LSPR wavelength were pulled out at different times.

The absorption spectra were measured using a UV-VISNIR spectrophotometer (Varian Cary 5000). The NLA and NLR of the samples were measured by Z-scan methods. The excitation source was a mode-locked Ti/sapphire pulsed laser (Mira 900, Coherent) with pulse width approximately $150 \mathrm{fs}$ and a repetition rate of $76 \mathrm{MHz}$. The optical length of the sample is $1 \mathrm{~mm}$.

\section{Results and Discussion}

Figure 1 shows the absorption spectra of the silver nanoplates with different sizes. They are characterized by the linear absorption spectra during the growth of the silver nanoplates at different times. There are two in-plane dipolar and quadrupolar plasmon modes of silver nanoplates. The dipolar resonance is extremely sensitive to the height and edge length of the silver nanoplates (the peak from 650 to $950 \mathrm{~nm})$. The quadrupolar resonance is weaker and also presents smaller red-shift compared to the dipole resonance.
A third plasmon resonance around $330 \mathrm{~nm}$ without shifting, which is contributed to the out-of-plane excitation, is also observed. The LSPR linearly red-shift and the intensity increased as the growth time goes by. While the full width at half maximum decreases with the growth time, which indicates a more uniform size of silver nanoplates as the growth time. The inserts of figure 1 present the two typical SEM images of the silver nanoplate with LSPR 650 and 950 $\mathrm{nm}$, respectively. We can observe that the silver nanoplates with LSPR $950 \mathrm{~nm}$ display larger edge length and more uniform size distribution.

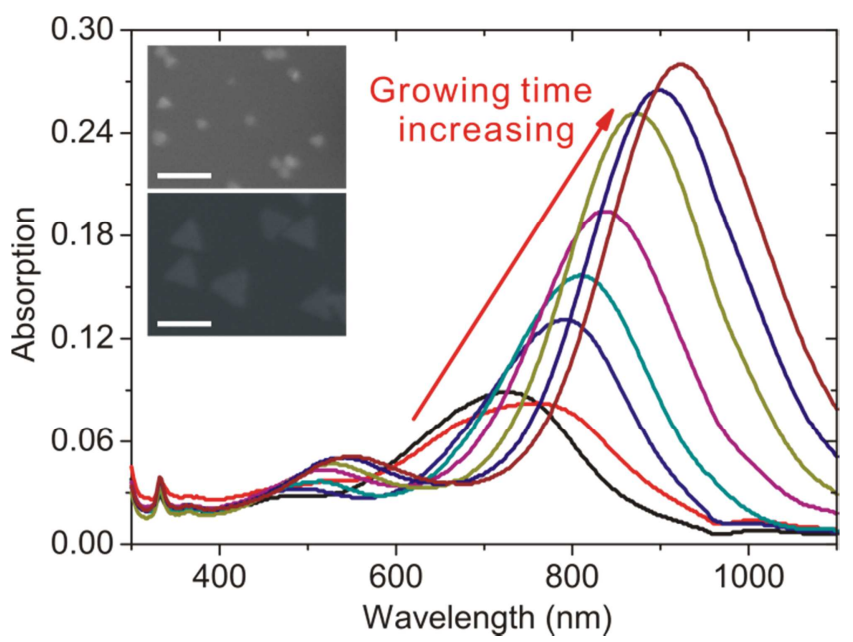

Figure 1. The absorption spectra of the silver nanoplates with different growth time. The insert presents the SEM images of the silver nanoplates with LSPR $650 \mathrm{~nm}$ and $950 \mathrm{~nm}$, respectively. The ruler bar is $200 \mathrm{~nm}$.
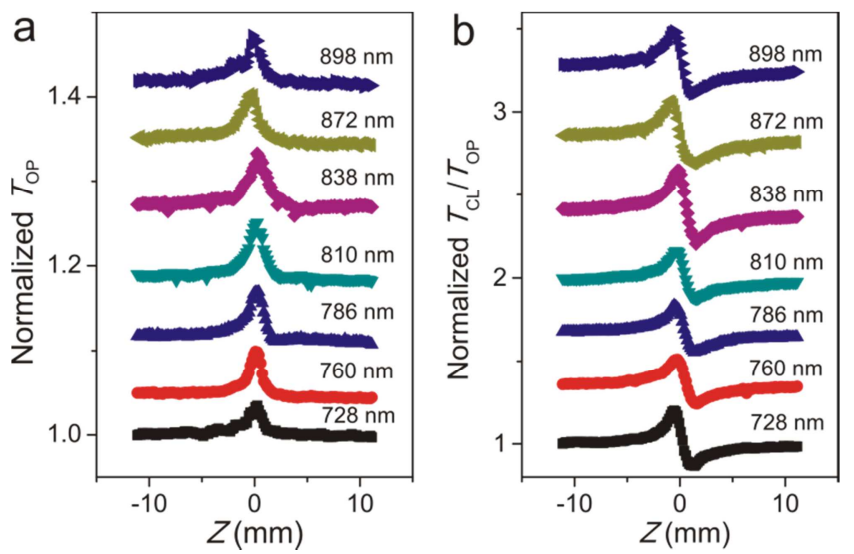

Figure 2. Normalized open aperture transmittance $T_{O P}$ (a) and close aperture $T_{C L} / T_{O P}$ curves (b) at the corresponding resonance wavelength of silver nanoplates with different sizes. The curves are shifted vertically for clarity.

Figure 2a shows the normalized open aperture Z-scan response of the silver nanoplates in water suspensions at corresponding longitude resonance wavelength varied from $728 \mathrm{~nm}$ to $898 \mathrm{~nm}$. When the light intensity $I$ is saturated, the power-dependent absorption coefficient $\alpha$ and refractive index $\mathrm{n}$ of materials could be described as $\alpha(I)=\alpha_{0}+\beta I$ and $n(I)=n_{0}+\gamma I$, where $\alpha_{0}$ and $n_{0}$ are the linear absorption coefficient and refractive index, respectively. In 
the Z-scan theory, the effective NLA coefficient $\beta$ and the NLR index $\gamma$ can be calculated by open aperture transmittance $T_{O P}$ and close aperture transmittance $T_{C L}$ as following relationships:

$$
\begin{aligned}
& T_{\mathrm{OP}}=\sum_{m=0}^{\infty} \frac{\left(-q_{0}\right)^{m}}{\left(1+z^{2} / z_{0}^{2}\right)^{m}(1+m)^{3 / 2}} \\
& \frac{T_{\mathrm{CL}}}{T_{\mathrm{OP}}}=1+\frac{4 \Delta \phi_{0} z / z_{0}}{\left(z^{2} / z_{0}^{2}+9\right)\left(z^{2} / z_{0}^{2}+1\right)}
\end{aligned}
$$

where $q_{0}=\beta_{\text {eff }} I_{0} L_{\text {eff }}$ and $\Delta \varphi_{0}=\gamma k I_{0} L_{\text {eff }}, I_{0}$ is the peak irradiance at the focus $(z=0), k=2 \pi / \lambda$ is the wave vector of the laser radiation and $z_{0}$ is the Rayleigh length of the Gaussian incident beam for typical two photon absorption (TPA) process. $L_{\text {eff }}=\left[1-\exp \left(-\alpha_{0} L\right)\right] / \alpha_{0}$ is the effective thickness of the samples, with $L$ being the length of the sample. As the sample moved into the focused beam, the transmittance is found to increase, which shows an optically induce transparency. The saturated absorption (SA) behavior

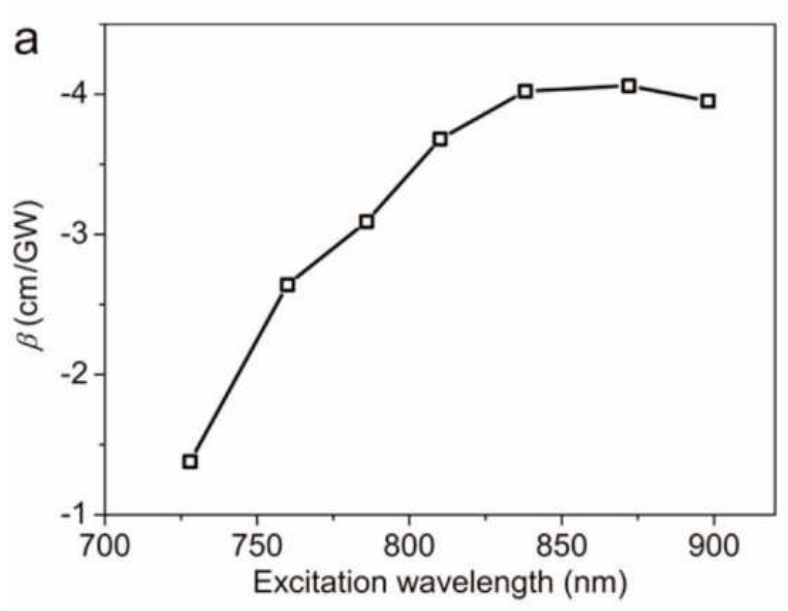

is attributed to the strong ground state plasmon bleaching. Figure $2 \mathrm{~b}$ presents the normalized $T_{C L} / T_{O P}$ curves of the samples, the saturated phenomenon is also obviously observed. And the NLR index $\gamma$ can be calculated by using the equation (2).

The third-order NLA coefficient $\beta$ and NLR index $\gamma$ of silver nanoplates are calculated, and the values are plotted as a function of excitation wavelength in figure 3 . The absolute value of effective NLA coefficient $|\beta|$ is increased from 1.38 to $4 \mathrm{~cm} / \mathrm{GW}$ (figure $3 \mathrm{a}$ ). Figure $3 \mathrm{~b}$ presents the tendency of NLR index $|\gamma|$ with different excitation wavelengths. With the increase of excitation wavelength from $728 \mathrm{~nm}$ to 898 $\mathrm{nm}$, the absolute value of NLR index $|\gamma|$ rises from 1.4 to $3.71 \mathrm{~cm}^{2} / \mathrm{GW}$. The increase absolute value of the NLR and NLA coefficients for different silver nanoplates attributes to the strong plasmon absorption and local filed enhancement of larger size silver nanoplates. Further studies are constructing to extent the LSPR of the samples to find the saturated absorption point since their higher aspect ratio between the two in-plane dipolar and the quadrupolar plasmon modes.

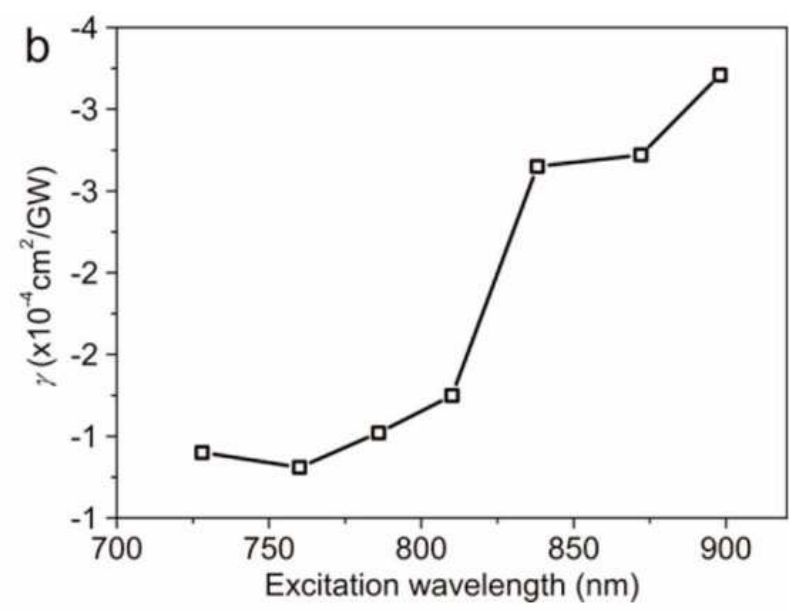

Figure 3. Third-order NLA coefficient $\beta$ (a) and NLR index $\gamma$ (b) of silver nanoplates plotted as a function of the corresponding resonant excitation wavelength.

\section{Conclusions}

In conclusion, we adopt a seed-mediated synthesis method to fabricate silver nanoplates with widely tunable LSPR ranging from $650 \mathrm{~nm}$ to $950 \mathrm{~nm}$. Moreover, we give a system investigation on the nonlinear absorption and refraction spectra dependence on the LSPR of the silver nanoplates. The absolute value of effective NLA coefficient $|\beta|$ is increased from 1.38 to $4 \mathrm{~cm} / \mathrm{GW}$. And the absolute value of NLR index $|\gamma|$ rises from 1.4 to $3.71 \mathrm{~cm}^{2} / \mathrm{GW}$. These findings will have potential applications in the biology sensors, solar cells and many other areas.

\section{Acknowledgements}

The project was supported by National Natural Science
Foundation of China (61701532).

\section{References}

[1] Wang, Y. Zhou, X. Wang, T. and Zhou, J. (2008). Enhanced luminescence from lanthanide complex by silver nanoparticles. Materials Letters, 62(20), 582. doi: 10.1016/j.matlet.2008.04.005.

[2] Ttebayashi, J. Yamada, T. Inaba, T. Timmerman, D. and Ichikawa, S. and Fujiwara, Y. (2019). Localized-surfaceplasmon-enhanced GaN:Eu-based red light-emitting diodes utilizing silver nanoparticles. Applied Physics Express, 12(9), 095003. doi: 10.7567/1882-0786/ab37b0.

[3] Okamoto, K. Niki, I. Shvartser, A. Narukawa, Y. Mukai, T. and Scherer, A. (2004). Surface-plasmon-enhanced light emitters based on InGaN quantum wells. Nature Materials, 3(9), 601. doi: 10.1038/nmat1198. 
[4] Lu, Z. Si, H. Li, Z. Yu, J. Liu, Y. Feng, D. Zhang, C. Yang, W. Man, B. and Jiang, S. (2018). Sensitive, reproducible, and stable 3D plasmonic hybrids with bilayer WS2 as nanospacer for SERS analysis. Optical Express, 26 (17), 21626. doi: 10.1364/OE.26.021626.

[5] Lu, Z. Liu, Y. Wang, M. Zhang, C. Li, Z. Huo, Y. Li, Z. Xu, S. Man, B. and Jiang, S. (2018). A novel natural surfaceenhanced Raman spectroscopy (SERS) substrate based on graphene oxide-Ag nanoparticles-Mytilus coruscus hybrid system. Sensors and Actuators B: Chemical, 261 (15), 1. doi: 10.1016/j.snb.2018.01.113.

[6] Bao, X. Liu, S. Wang, Y. and Zhao, L. (2011). Enhanced light absorption of $\mathrm{Ag}$ films deposited onto femtosecond laser microstructured silicon. Materials Letters, 65 (12), 1927. doi: 10.1016/j.matlet.2011.03.018.

[7] Wang, Y. Liu, S. Wang, Y. Feng, G. Zhu, J. and Zhao, L. (2009). Infrared light absorption of silver film coated on the surface of femtosecond laser microstructured silicon in SF6. Materials Letters, 63 (30), 2718. doi: 10.1016/j.matlet.2009. 09.052 .

[8] Jain, P. K. Lee, K. S. El-Sayed, I. H. and El-Sayed, M. A. (2006). Calculated Absorption and Scattering Properties of Gold Nanoparticles of Different Size, Shape, and Composition: Applications in Biological Imaging and Biomedicine. The Journal of Physical Chemistry B, 110 (14), 7238. doi: $10.1021 /$ jp057170o.

[9] Kelly, K. L. Coronado, E. Zhao, L. L. and Schatz, G. C. (2003). The Optical Properties of Metal Nanoparticles: The Influence of Size, Shape, and Dielectric Environment. The Journal of Physical Chemistry B, 107 (3), 668. doi: 10.1021/jp026731y.

[10] Khan, I. Saeed, K. and Khan, I. (2019). Nanoparticles: Properties, applications and toxicities. Arabian Journal of Chemistry, 12 (7), 908. doi: 10.1016/j.arabjc.2017.05.011.

[11] Hu, G. Jin, W. Zhang, W. Wu, K. He, J. Zhang, Y. Chen, Q. and Zhang, W. (2018). Surfactant-assisted shape separation from silver nanoparticles prepared by a seed-mediated method. Colloids and Surfaces A: Physicochemical and Engineering Aspects, 540 (5), $136 . \quad$ doi: 10.1016/j.colsurfa.2017.12.071.

[12] Goswami, S. Noh, H. Redfern, L. R. Otake, K.-I. Kung, C.-W. Cui, Y. Chapman, K. W. Farha, O. K. and Hupp, J. T. (2019). Pore-Templated Growth of Catalytically Active Gold Nanoparticles within a Metal-Organic Framework. Chemistry of Materials, 31 (5), $1485 . \quad$ doi: 10.1021/acs.chemmater.8b04983.

[13] Yang, L. Zhou, Z. Song, J. and Chen, X. (2019). Anisotropic nanomaterials for shape-dependent physicochemical and biomedical applications. Chemical Society Reviews, 48 (19), 5140. doi: 10.1039/C9CS00011A.

[14] Chen, S. Fan, Z. and Carroll, D. L. (2002). Silver Nanodisks: Synthesis, Characterization, and Self-Assembly. The Journal of Physical Chemistry B, 106 (42), 10777. doi: 10.1021/jp026376b.

[15] Kondorskiy, A. D. Lam, N. T. and Lebedev, V. S. (2018). Absorption and Scattering of Light by Silver and Gold Nanodisks and Nanoprisms. Journal of Russian Laser Research, 39 (1), 56. doi: 10.1007/s10946-018-9689-1.

[16] Krishnan, S. K. Esparza, R. Flores-Ruiz, F. J. Padilla-Ortega,
E. Luna-Bárcenas, G. Sanchez, I. C. and Pal, U. (2018). SeedMediated Growth of Ag@Au Nanodisks with Improved Chemical Stability and Surface-Enhanced Raman Scattering. ACS Omega, 3 (10), 12600. doi: 10.1021/acsomega.8b02333.

[17] Bi, Y. and Lu, G. (2008). Morphological controlled synthesis and catalytic activities of gold nanocrystals. Materials Letters, 62 (17), 2696. doi: 10.1016/j.matlet.2008.01.021.

[18] Luo, Y. (2007). Large-scale preparation of single-crystalline gold nanoplates. Materials Letters, 61 (6), 1346. doi: 10.1016/j.matlet.2006.07.048.

[19] Zeng, J. Zheng, Y. Rycenga, M. Tao, J. Li, Z.-Y. Zhang, Q. Zhu, Y. and Xia, Y. (2010). Controlling the Shapes of Silver Nanocrystals with Different Capping Agents. Journal of the American Chemical Society, 132 (25), 8552. doi: $10.1021 /$ ja103655f.

[20] Rycenga, M. Cobley, C. M. Zeng, J. Li, W. Moran, C. H. Zhang, Q. Qin, D. and Xia, Y. (2011). Controlling the Synthesis and Assembly of Silver Nanostructures for Plasmonic Applications. Chemical Reviews, 111 (6), 3669. doi: $10.1021 / \operatorname{cr} 100275 \mathrm{~d}$

[21] Ning, R. Lu, W. Zhang, Y. Qin, X. Luo, Y. Hu, J. Asiri, A. M. Al-Youbi, A. O. and Sun, X. (2012). A novel strategy to synthesize Au nanoplates and their application for enzymeless $\mathrm{H} 2 \mathrm{O} 2$ detection. Electrochimica Acta, 60 (15), 13. doi: 10.1016/j.electacta.2011.10.066.

[22] Wenbo, L. Yonglan, L. Guohui, C. Abdullah, M. A. Abdulrahman, O. a.-Y. and Xuping, S. (2012). Supramolecular Microfibrils of O-Phenylenediamine Dimers: Oxidationinduced Formation of $\mathrm{Au}$ Nanoparticle-decorated Nanoplates for $\mathrm{H} 2 \mathrm{O} 2$ Detection. Current Nanoscience, 8 (2), 221. doi: $10.2174 / 157341312800167696$.

[23] Tsang, H. K. Wong, C. S. Liang, T. K. Day, I. E. Roberts, S. W. Harpin, A. Drake, J. and Asghari, M. (2002). Optical dispersion, two-photon absorption and self-phase modulation in silicon waveguides at $1.5 \mu \mathrm{m}$ wavelength. Applied Physics Letters, 80 (3), 416. doi: 10.1063/1.1435801.

[24] Jagannath, G. Eraiah, B. Nagakrishnakanth, K. and Venugopal, Rao S. (2018). Linear and nonlinear optical properties of gold nanoparticles doped borate glasses [J]. Journal of Non-Crystalline Solids, 482 (15), 160. doi: 10.1016/j.jnoncrysol.2017.12.036.

[25] Jiang, Y. Ma, Y. Fan, Z. et al. (2018). Abnormal nonlinear optical properties of hybrid graphene-TiO 2 nanostructures. Optics letters, 43 (3), 523. doi: 10.1364/OL.43.000523.

[26] DeSalvo, R. Sheik-Bahae, M. Said, A. A. Hagan, D. J. and Van Stryland, W. (1993). Z-scan measurements of the anisotropy of nonlinear refraction and absorption in crystals. Optics letters, 18 (3), 194. doi: 10.1364/OL.18.000194.

[27] Hussain, M. S. Hassan, Q. M. A. Sultan, H. A. Al-Asadi, A. S. Chayed, H. T. and Emshary, C. A. (2019). Preparation, characterization, and study of the nonlinear optical properties of a new prepared nanoparticles copolymer. Modern Physics Letters B, $33 \quad$ (36), $1950456 . \quad$ doi: $10.1142 / \mathrm{S} 0217984919504566$.

[28] Smith, D. D. Yoon, Y. Boyd, R. W. Campbell, J. K. Baker, L. A. Crooks, R. M. and George, M. (1999). z-scan measurement of the nonlinear absorption of a thin gold film. Journal of Applied Physics, 86 (11), 6200. doi: 10.1063/1.371675. 
[29] Sederberg, S. Firby, C. J. and Elezzabi, A. Y. (2019). Efficient, broadband third-harmonic generation in silicon nanophotonic waveguides spectrally shaped by nonlinear propagation. Optics express, 27 (4), 4990. doi: 10.1364/OE.27.004990.

[30] Jia, Y. Liao, Y. Wu, L. Shan, Y. Dai, X. Cai, H. Xiang, Y. and Fan, D. (2019). Nonlinear optical response, all optical switching, and all optical information conversion in $\mathrm{NbSe} 2$ nanosheets based on spatial self-phase modulation. Nanoscale, 11 (10), 4515. doi: 10.1039/C8NR08966C.

[31] Kochuparampil, A. P. Joshi, J. H. Jethva, H. O. and Joshi, M. J. (2019). Modification in structural, functional, thermal, dielectric, impedance and nonlinear optical properties of ammonium pentaborate crystal by addition of di-sodium sulphide nanoparticles. Modern Physics Letters B, 33 (07), 1950084. doi: 10.1142/S0217984919500842.

[32] Wu, L. F. Wang, Y. H. Li, P. L. Wu, X. Shang, M. Xiong, Z. Z. Zhang, H. J. Liang, F. Xie, Y. F. and Wang, J. (2019). Enhanced nonlinear optical behavior of graphene-CuO nanocomposites investigated by Z-scan technique. Journal of Alloys and Compounds, 777 (10), 759. doi: 10.1016/j.jallcom.2018.11.045.

[33] Krishnakanth, K. N. Seth, S. Samanta, A. and Rao, S. V. (2018). Broadband femtosecond nonlinear optical properties of $\mathrm{CsPbBr} 3$ perovskite nanocrystals. Optics Letters, 43 (3), 603. doi: 10.1364/OL.43.000603. 